\title{
The dynamic transition in proteins may have a simple explanation
}

\author{
Roy M. Daniel, ${ }^{a}$ John L. Finney ${ }^{b}$ and Jeremy C. Smith ${ }^{c}$ \\ ${ }^{a}$ Department of Biological Sciences, University of Waikato, Hamilton, New Zealand \\ ${ }^{b}$ Department of Physics and Astronomy, University College London, Gower Street, \\ London, UK WCIE 6BT \\ ${ }^{c}$ Lehrstuhl für Biocomputing, IWR, Universität Heidelberg, Im Neuenheimer Feld 368, \\ D-69120 , Heidelberg, Germany
}

\author{
Received 25th January 2002, Accepted 19th March 2002 \\ First published as an Advance Article on the web 3rd July 2002
}

The transition that has been observed in the dynamics of hydrated proteins at low temperatures $(180-230 \mathrm{~K})$ is normally interpreted as a change from vibrational, harmonic motion at low temperatures to anharmonic motions as the temperature is raised. It is taken to be an intrinsic property of proteins and has been associated with the onset of protein functions. Examination of the dynamic behaviour of proteins in solution within a defined timescale window suggests that certain observations can be explained without the need to invoke a discontinuity in the dynamics of proteins with temperature, i.e. the existence of a dynamical transition is not required. This is discussed in the context of recent evidence that enzyme activity is independent of the activation of anharmonic picosecond dynamics and declines steadily with temperature through the apparent dynamic transition, in accordance with the Arrhenius relationship. That similar timescale dependent dynamical behaviour has been observed experimentally in chain polymers, and seen also in computer simulations of silica glasses, suggests that the phenomenon may be of wide general relevance in both simple glassy and more complex polymeric systems.

\section{Introduction}

Protein function involves structural changes, sometimes small and barely detectable, and sometimes involving significant, correlated motions over relatively large length scales. Determining which motions exist in proteins and which of these are required for function is thus a fundamental challenge.

Several techniques have shown a transition in the dynamics of hydrated proteins at low temperatures. ${ }^{1-16}$ Much of this work has been done on myoglobin using Mössbauer spectroscopy ${ }^{3,6,8}$ neutron scattering ${ }^{10,11}$ or X-ray crystallography ${ }^{2}$ of hydrated crystals, powders, or frozen solutions, but similar results have been found in X-ray crystallographic studies of ribonuclease $\mathrm{A}^{12}$ and in Mössbauer ${ }^{14}$ and neutron scattering ${ }^{15}$ studies of membrane proteins. Some protein functions have been observed to cease with the loss of equilibrium anharmonic dynamics as the protein is cooled through the dynamic transition. Among these are electron tunnelling in Rhodospirullum rubrum chromatophores, ${ }^{14}$ some elements of the photocycle of bacteriorhodopsin in hydrated membranes of Halobacterium salinarum, ${ }^{15}$ and ligand binding/release in ribonuclease A crystals. ${ }^{16}$

This discontinuity is interpreted as a transition from vibrational, harmonic motion at low temperatures to anharmonic motions as the temperature is raised. Most experimental results arise 
from studies of hydrated protein powders, or crystals, using a range of techniques. These include Mössbauer spectroscopy of the Fe ion in myoglobin, X-ray scattering measurements of the temperature factor in protein crystals, Rayleigh scattering of Mössbauer radiation, and neutron scattering to probe the global dynamics of a relatively limited number of proteins. ${ }^{1-16}$ Depending on the technique and possibly the protein or the nature of the preparation, the sharpness and temperature of this transition may vary somewhat, but has been generally observed between about 180 and $230 \mathrm{~K}$. It is taken to be an intrinsic property of proteins and, as indicated above, has been associated with the onset of protein function. ${ }^{14-20}$

Recent neutron scattering measurements on a thermophilic glutamate dehydrogenase enzyme in solution, under conditions in which enzyme activity is both possible and measurable, failed to show any relationship between an observed dynamical transition at around $220 \mathrm{~K}$ and the onset of activity $^{13}$ (Fig. 1). The activity was observed to maintain an Arrhenius temperature dependence to well below this temperature, indicating that the transition observed in this system has no effect on the catalytic activity of this soluble, multisubunit enzyme. Results from a similar study carried out on a xylanase enzyme suggest that the behaviour of single subunit enzymes are also unaffected by the observed transition, although activity measurements were made here down to only $200 \mathrm{~K} .{ }^{21} \mathrm{We}$ have now measured activity in a number of enzymes between $200 \mathrm{~K}$ and $170 \mathrm{~K},{ }^{22}$ and there is so far no evidence for any intrinsic lower temperature limit for enzyme activity, although the lowest temperature at which enzyme activity has been measured to date is $170 \mathrm{~K}^{22}$ The association of the dynamical transition with the onset of enzyme activity is thus brought into question.

We need however to be aware of a number of points in considering the possible relationship between enzyme activity and dynamics. For example, there is the possible dependence of the results on the experimental or computational probe used. Diffraction methods such as X-ray crystallography on protein crystals produce time-averaged information via temperature factors. Due to their time-averaged nature, these results also contain static disorder effects. Other techniques such as Mössbauer spectroscopy of fluorescence depolarisation measure localised signals on specific timescales. Neutron scattering is a probe of faster motions (ps to ns depending on the instrument used) of mainly the hydrogen atoms in the system, and is hence a probe of global dynamics of the molecule. Finally molecular dynamics simulation can provide detailed modelling results on either global or localised dynamics over timescales stretching from ps to ns. Furthermore, we need to be sensitive to the environment of the proteins examined. For good experimental reasons, dynamical transition measurements have been made in the main on proteins under conditions of low hydration or in lipoprotein membranes rather than in the solution conditions in which the protein

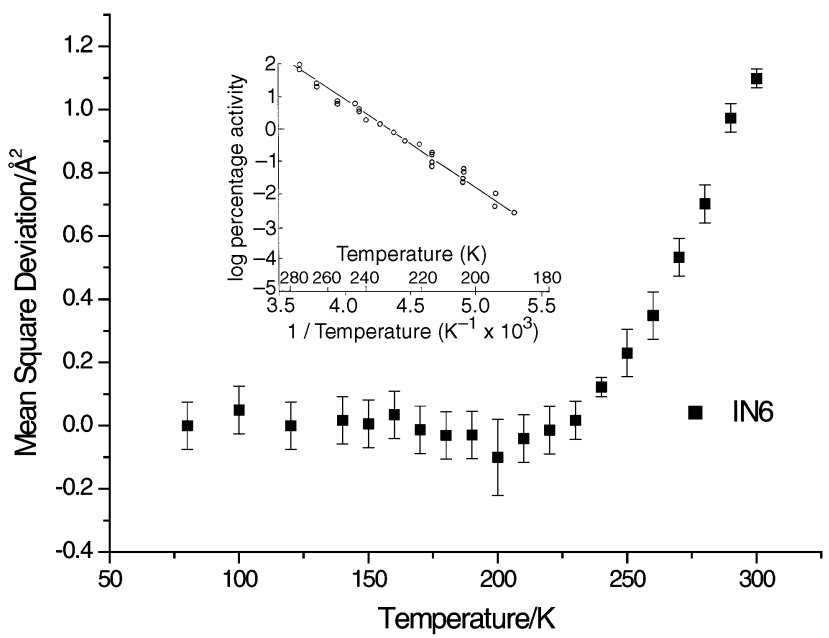

Fig. 1 Mean square displacement, measured on IN6, of GDH in 70\% methanol in water, as a function of temperature $T$. The inset shows the activity of this enzyme under the same conditions, which is seen to be Arrhenius over the whole temperature range covered. 
is normally active. Hence our insistence, in the parallel activity/dynamics measurements mentioned above, on performing the dynamics measurements under the same solvent conditions as those under which the activity measurements are made. The variation of dynamics with solvent conditions is therefore in itself a relevant area of study. ${ }^{23}$

We focus here on the possible dependence of the dynamical transition on the timescale of the motions probed. For such measurements, neutron scattering is a particularly useful technique, as the timescale of the motions measured depends on the resolution of the instrument used. As the sample requirements are similar for different resolution instruments, the dynamics of the same system under the same environmental conditions can be probed using different timescale windows. In the results given below, we use the two instruments IN6 and IN16 at the Institut Laue-Langevin, Grenoble, France. IN6 probes motions in the timescale faster than about 100 ps, while the motions reported by IN16 are faster than about 5000 ps.

\section{Methods}

The glutamate dehydrogenase enzyme used is from Thermococcus strain AN1 (now known as T. zilligii strain AN1) (DSM 2770), and was purified, assayed and prepared for neutron scattering as described elsewhere. ${ }^{13,24,25}$ The xylanase enzyme was obtained from an E. coli clone containing the gene from the extremely thermophilic bacterium Thermotoga maritima strain FjSS3-B.1. ${ }^{21,26}$ Its purification, assay and preparation for neutron scattering are described elsewhere. ${ }^{22}$

The neutron scattering measurements were performed on the IN6 time-of-flight spectrometer and on the IN16 backscattering spectrometer at the Institut Laue-Langevin, Grenoble. The incident neutron wavelengths were $5.12 \AA$ on IN6 and $6.28 \AA$ on IN16. All data were collected with the sample holder oriented at $135^{\circ}$ relative to the incident beam. The samples were contained in aluminium flat-plate cells, of $0.3 \mathrm{~mm}$ and $0.5 \mathrm{~mm}$ path length on IN6 and IN16 respectively.

Samples were of between 50 and $100 \mathrm{mg} \mathrm{ml}^{-1}$ of enzyme in $70 \% \mathrm{v} / \mathrm{v} \mathrm{CD} 3 \mathrm{OD} / \mathrm{D}_{2} \mathrm{O}$ solvent. The samples were cooled to $80 \mathrm{~K}$ then heated progressively to $320 \mathrm{~K}$ over 16-24 h. Raw data on the two instruments were corrected in identical fashion. The elastic intensity was determined by integrating the detector counts over the energy range of the instrumental resolution. The detectors were calibrated by normalising with respect to a standard vanadium sample. The cell scattering was subtracted, taking into account attenuation of the singly scattered beam. Finally, the scattering was normalised with respect to the scattering at the lowest measured temperature, $80 \mathrm{~K}$, and to the lowest wave vector $q$.

The elastic incoherent scattering intensity $S_{\text {inc }}(q, \omega=0)$ (where $q$ is the magnitude of the scattering wave vector and $\omega$ is the energy transfer) was used to obtain the average mean square displacement $\left\langle u^{2}\right\rangle$ using the relationship $\ln S_{\text {inc }}(q, \omega=0)=-\left\langle u^{2}\right\rangle q^{2} / 3$ which is valid in the regime $q^{2}\left\langle u^{2}\right\rangle / 3<1$. $\left\langle u^{2}\right\rangle$ was thus obtained by fitting a straight line to a semi-log plot of $S(q, \omega=0)$ versus $q^{2}$ in the linear regime which was found at $0.12 \AA^{-2}<q^{2}<1.07 \AA^{-2}$ and $0.10 \AA^{-2}<q^{2}<1.13$ $\AA^{-2}$ in the IN6 and IN16 experiments, respectively. The linear regime was found to be wellseparated from the Bragg scattering of the solution which was found at $1.4 \AA^{-1}<q<2.0 \AA^{-1}$, and no evidence was found for a low- $q$ protein-protein interaction peak. As the scattering was normalised with respect to the $80 \mathrm{~K}$ intensities, the $\left\langle u^{2}\right\rangle$ determined is equal to $\left(\left\langle u^{2}\right\rangle_{T}-\left\langle u^{2}\right\rangle_{80}\right)$ where $\left\langle u^{2}\right\rangle_{T}$ is the absolute mean-square displacement at temperature $T$. In practice, the measured $\left\langle u^{2}\right\rangle$ corresponds to the $\mathrm{H}$ atoms, whose scattering cross section is strongly dominant. Depending on the concentration of the sample, between $70 \%$ and $80 \%$ of the incoherent signal is due to the enzyme, respectively. The $\left\langle u^{2}\right\rangle$ obtained for these samples are therefore dominated by the enzyme motions. The energy resolution of IN16 is $1 \mu \mathrm{eV}$ whereas that of IN6 is $50 \mu \mathrm{eV}$. The inverses of these energy resolutions correspond to times of $5 \mathrm{~ns}$ and $100 \mathrm{ps}$, respectively.

\section{Results}

\section{Glutamate dehydrogenase}

The temperature dependence of the rms displacement of this multisubunit (hexameric) enzyme in $70 \%$ methanol (the cryosolvent in which activity measurements have been made at low temperature $^{24}$ ) is given in Fig. 2 for the two different instruments. There is a clear indication from Fig. 2 of 


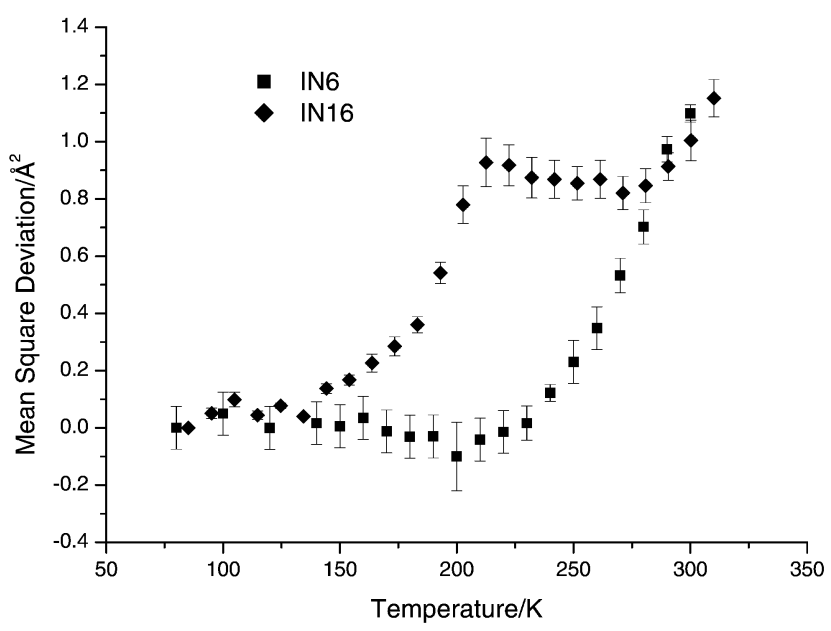

Fig. 2 Mean square displacement of GDH in $70 \%$ methanol in water, as a function of temperature $T$, measured on both IN6 and IN16.

a timescale-dependence of this dynamical transition. The onset of anharmonic motion occurs at $\sim 140 \mathrm{~K}$ on IN16 (motions $<\sim 5 \mathrm{~ns}$ ), and $\sim 220 \mathrm{~K}$ on IN6 ( $<\sim 100 \mathrm{ps}$ ). Further measurements on a spectrometer of intermediate resolution (IRIS at the ISIS pulsed spallation neutron source, Rutherford Appleton Laboratory, UK) shows a dynamic transition at an intermediate temperature. ${ }^{28}$ Referring to the IN16 data in Fig. 2, in addition to the dynamical transition at $\sim 140 \mathrm{~K}$, there are inflections in the IN16 $\left\langle u^{2}\right\rangle$ at $\sim 185 \mathrm{~K}, \sim 210 \mathrm{~K}$ and $\sim 280 \mathrm{~K}$. If one defines a 'dynamical transition' as an inflection in $\left\langle u^{2}\right\rangle$, then the IN16 profile demonstrates the presence of four dynamical transitions in the sample. The three highest-temperature transitions do not correspond to transitions from anharmonic to harmonic behaviour, rather to modification of the anharmonic behaviour itself. Focussing on the lowest transition at each timescale, this result implies that, for this protein solution, the temperature of the observed onset of anharmonic motion does indeed depend on the timescale of the motions explored ( $\sim 100 \mathrm{ps}$ and $\sim 5 \mathrm{~ns}$ in Fig. 2). Parallel differential scanning calorimetry and synchrotron X-ray powder diffraction measurements on both the enzyme preparations and the pure cryosolvent confirm ${ }^{27}$ that the lowest transitions measured on each instrument are not related to phase changes in the solvent.

\section{Xylanase}

Because of the possibility that this initially surprising result might hold only for a multisubunit enzyme, where we might perhaps expect longer timescale motions to relate to subunit motions, we have recently made a series of similar measurements on the smaller, single subunit enzyme xylanase, in the same solvent. ${ }^{21,29}$ The mean square displacement as a function of temperature measured on the two different instruments is shown in Fig. 3. The trend of the results is similar to that found for glutamate dehydrogenase (Fig. 2), confirming that the temperature of the observed onset of anharmonic motion depends on the timescale of the motions explored, and that this conclusion is valid for both the hexameric and single subunit enzymes examined.

\section{Discussion}

We recall that IN6 probes motions faster than about $100 \mathrm{ps,} \mathrm{while} \mathrm{IN16} \mathrm{covers} \mathrm{a} \mathrm{wider} \mathrm{range} \mathrm{up} \mathrm{to}$ $5 \mathrm{~ns}$. If we therefore subtract these two data sets for the same sample, we will obtain information on motions of that sample within a timescale window delimited by $100 \mathrm{ps}$ at the faster end and $5000 \mathrm{ps}$ at the slower end. The difference will therefore show the temperature dependent behaviour of enzyme motions within a defined timescale window of between 100 and 5000 ps. The subtraction also has the advantage of largely removing the residual $20-30 \%$ contribution of the solvent motions from the data. The results of this subtraction for the two enzymes are shown in Fig. 4. 


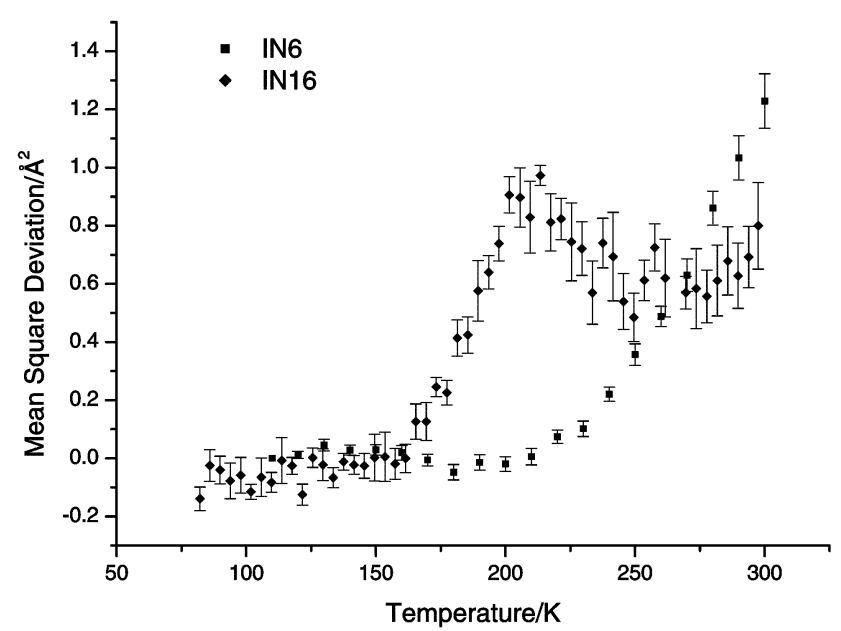

Fig. 3 Mean square displacement of xylanase in $70 \%$ methanol in water, as a function of temperature $T$, measured on both IN6 and IN16.

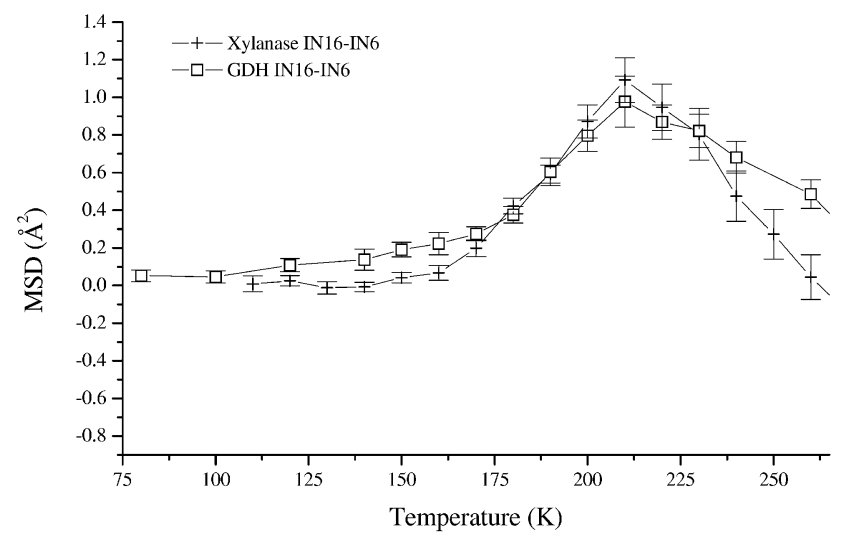

Fig. 4 Protein global dynamics in a 100 to 5000 ps timescale window. This figure shows the subtraction of the average mean square displacements of the protons observed up to $100 \mathrm{ps}$ (instrument IN6) from those observed up to 5000 ps (instrument IN16), for xylanase and glutamate dehydrogenase in $70 \%$ methanol; i.e. the difference plot shows the motions for each protein within a timescale window of 100 to $5000 \mathrm{ps}$. The data used is that presented in Figs. 2 and 3.

Within the experimental uncertainties, Fig. 4 shows that the difference results for the two (very different) enzymes are remarkably similar, giving a roughly symmetrical peak at around $240 \mathrm{~K}$. At the lowest temperatures, no motions faster than the slowest motions that will be visible in this window (characteristic times of around $5000 \mathrm{ps}$ ) are observed. As the temperature is raised above about $160 \mathrm{~K}$, the slowest motions towards the observational limit of $5000 \mathrm{ps}$ are detected. As temperature is increased further, faster (higher energy) motions are excited, and begin to contribute to the increase in observed mean square displacements. With a further temperature increase the mean square displacements decline, as the frequencies increase further and pass out of the 100 to 5000 ps timescale window.

This behaviour is consistent with that of simple activated dynamics, observed through a defined timescale window. A key distinguishing feature of a discontinuity arising from such behaviour would be its dependence on the instrument timescale. The implication of timescale dependence is that if the dynamics gradually and continuously slow as the temperature decreases, then any technique which observed only a part of the dynamic timescale would record a transition in the 
dynamics at the point where it could no longer record the motions because they were too slow. Thus, in this simplest interpretation of the observed timescale dependence, the existence of a dynamical transition is not necessary to explain the experimental results to date.

We now conduct thought experiments on what might be seen using two spectrometers of different energy resolution. Three scenarios may be considered. In the first there is a change in the long-time (equilibrium) dynamics of the enzyme system, and both spectrometers are considered to be of high enough resolution to resolve the motions concerned at all temperatures. In this case there will be a transition in the data that is independent of instrumental resolution. In the second scenario there is no change in the long-time dynamics of the enzyme system with temperature but the characteristic relaxation times pass into the resolved energy window of each instrument as the temperature increases. In this case a transition will also be observed in the data, and this will be dependent on instrumental resolution. The third scenario is similar to the first in that there is a change in the long time dynamics of the enzyme system, but differs from the first in that the instrumental resolutions are such that not all motions are resolved at all temperatures. Depending on the resolutions of the instruments with respect to the enzyme system dynamics, this could also lead to a temperature-dependent transition behaviour.

The first scenario is somewhat theoretical in that the spectrometers used are unlikely to be of high enough resolution to fulfil the stated conditions. Moreover, it is not consistent with the observed behaviour of our two enzyme solutions. Given the wide range of timescales in which motions in proteins exist, and X-ray diffraction evidence of a time-averaged (long-time) change in dynamics with temperature (but admittedly made more complex by the difficulty of separating out the effects of static disorder) it is possible that the third scenario could apply. If it does, then we have the problem of explaining why this dynamic transition has no effect on enzyme activity in solution, which is expected to be dependent on dynamics. However, the second scenario, which explains the observed temperature dependence of an apparent dynamical transition in terms of the resolution windows of the spectrometers used, is all that is necessary to explain the observed behaviour. It is the simplest explanation, and therefore passes the Occam's razor test. Moreover it does not require the existence of a dynamic transition, the observed behaviour being consistent with a simpler dynamical picture in which the motions of the enzyme in solution slow continuously with temperature, as the activity is observed to do.

The interpretation based on this second scenario clearly raises questions concerning the direct association observed in some experiments of a dynamic transition with the onset of protein function. ${ }^{14-20}$ The processes for which these associations have generally been made, such as electron transport or ligand binding, ${ }^{14-16}$ are those involving relatively fast reactions (and under conditions of relatively low hydration, rather than in solution). This is presumably because of a dependence upon the faster motions, which become too slow to allow normal function. Enzyme function however, usually occurring over millisecond timescales, is probably dependent upon slower motions, and thus likely to be independent of the activation of the picosecond-timescale dynamical transition observed in solution. This is consistent with our results that demonstrate conclusively that enzyme activity in solution is completely independent of any possible transition in picosecond motions: no departure from Arrhenius behaviour is observed down to the lowest temperature at which activity has been measured, $173 \mathrm{~K}^{13,21,22,24,27}$ Thus, if any observed dynamical transition is to be related to the onset of protein function, account must be taken of the timescales of the relevant processes. If there is indeed no dynamic transition, then our activity and dynamics results can be explained very simply on the basis of slower motions resulting in lower activity.

\section{Conclusions}

The absence of a relationship between the picosecond-timescale dynamic transition observed in solution and the onset of activity demonstrates clearly that at $220 \mathrm{~K}$ activated picosecond dynamics are not necessarily coupled to the motions that are important for the rate-limiting step of enzymes in solution. The lower temperature at which the nanosecond-timescale transition occurs in Figs. 2 and 3 may or may not be associated with the onset of enzyme activity; activity measurements at such low temperatures have not yet been possible. But it is clear that the simple association of a 
dynamical transition with enzyme activity cannot be made independent of a consideration of the timescale of the measured transition. The evidence to date is consistent with a model in which the motions of proteins in solution slow continuously with temperature. Thus the observed dynamical transition may be no more than the appearance of motions within the observational timescale window of the instrument used.

In conclusion, we note two further relevant points. First, similar timescale dependences of the dynamics of large molecules have also been made in neutron scattering measurements of chain polymers. ${ }^{30,31}$ They have also been seen in computer simulations of silica glasses. ${ }^{32}$ Thus, the behaviour discussed here may well be much more general than just its presence in enzymes in solution. Secondly, a recent theoretical model of Becker et al. ${ }^{33}$ shows that the kind of timescale dependence observed for the dynamics of these two enzymes can be reproduced if the characteristic relaxation frequencies of the protein are assumed to be temperature dependent. This model, which does not require a change in the time-averaged dynamics, gives good agreement with the experimental neutron scattering data discussed here.

\section{Acknowledgements}

We thank the Marsden Fund (NZ) and the Royal Society (UK) for financial support, and the Institut Laue-Langevin, Grenoble, and ISIS, Rutherford Appleton Laboratory, for the use of neutron beam facilities.

\section{References}

1 F. Parak and H. Formanek, Acta Crystallogr., Sect. A, 1971, 27, 573.

2 H. Frauenfelder, G. A. Petsko and D. Tsernoglou, Nature, 1979, 280, 558.

3 H. Keller and P. G. Debrunner, Phys. Rev. Lett., 1980, 45, 68.

4 F. Parak, E. N. Frolov, R. L. Mössbauer and V. I. Goldanskii, J. Mol. Biol., 1981, 145, 825.

5 S. G. Cohen, E. R. Bauminger, I. Nowik, S. Ofer and J. Yariv, Phys. Rev. Lett., 1980, 46, 1244.

6 E. W. Knapp, S. F. Fischer and F. Parak, J. Phys. Chem., 1982, 86, 5042.

7 H. Hartmann, F. Parak, W. Steigman, G. A. Petsko, D. R. Ponzi and H. Frauenfelder, Proc. Natl Acad. Sci. USA, 1982, 79, 4967.

8 F. Parak, E. W. Knapp and D. Kucheida, J. Mol. Biol., 1982, 161, 177.

9 E. R. Bauminger, S. G. Cohen, I. Nowik, S. Ofer and J. Yariv, Proc. Natl Acad. Sci. USA, 1983, 80, 736.

10 W. Doster, S. Cusack and W. Petry, Nature, 1989, 337, 754.

11 S. Cusack and W. Doster, Biophys. J., 1990, 58, 243.

12 R. F. Tilton, J. C. Dewan and G. A. Petsko, Biochemistry, 1992, 31, 2469.

13 R. M. Daniel, J. C. Smith, M. Ferrand, S. Hery, R. Dunn and J. L. Finney, Biophys. J., 1998, 75, 2504.

14 F. Parak, E. N. Frolov, A. A. Kononenko, R. L. Mössbauer, V. I. Goldanskii and A. G. Rubin, FEBS Lett., 1980, 117, 368.

15 M. Ferrand, A. J. Dianoux, W. Petry and G. Zaccai, Proc. Natl Acad. Sci. USA, 1993, 90, 9668.

16 B. F. Rassmussen, A. M. Stock, D. Ringe and G. A. Petsko, Nature, 1992, 357, 523.

17 J. Fitter, R. E. Lechner and N. Dencher, Biophys. J., 1997, 73, 2126.

18 U. Lehnert, V. Réat, M. Weil, G. Zaccai and C. Pfister, Biophys. J., 1998, 75, 1945.

19 X. Ding, B. F. Rasmussen, G. A. Petsko and D. Ringe, Biochemistry, 1994, 33, 9285.

20 A. Ostermann, R. Waschipky, F. G. Parak and G. U. Nienhaus, Nature, 2000, 404, 205.

21 R. V. Dunn, V. Réat, J. L. Finney, M. Ferrand, J. C. Smith and R. M. Daniel, Biochem. J., 2000, $346,355$.

22 J. M. Bragger, R. V. Dunn and R. M. Daniel, Biochim. Biophys. Acta, 2000, 1480, 278.

23 V. Réat, R. Dunn, M. Ferrand, J. L. Finney, R. M. Daniel and J. C. Smith, Proc. Natl Acad. Sci. USA, 2000, 97, 9961 .

24 N. More, R. M. Daniel and H. H. Petach, Biochem. J., 1995, 305, 17.

25 R. C. Hudson, L. D. Ruttersmith and R. M. Daniel, Biochim. Biophys. Acta, 1993, 1202, 244.

26 D. J. Saul, L. C. Williams, R. A. Reeves, M. D. Gibbs and P. L. Bergquist, Appl. Environ. Microbiol., 1995 , 61, 4110 .

27 R. M. Daniel, J. L. Finney, V. Réat, R. Dunn, M. Ferrand and J. C. Smith, Biophys. J., 1999, 77, 2184.

28 J. L. Finney and R. M. Daniel, ISIS Experimental Report 1998, Rutherford Appleton Laboratory, Chilton, Didcot, Oxon, UK, 1998, RB8790.

29 J. L. Finney, R. M. Daniel, J. C. Smith and V. Réat, ILL report on Expt 8-05-341, http://www.ill.fr/.

30 B. Frick and D. Richter, Science, 1995, 267, 1939.

31 C. A. Angell, Science, 1995, 267, 1924.

32 C. A. Angell, Comput. Mater. Sci., 1995, 4, 285.

33 T. Becker, J. L. Finney, R. M. Daniel and J. C. Smith, in preparation. 